\title{
How Common are Engines in Ib/c Supernovae?
}

\author{
Edo Berger \\ Division of Physics, Mathematics and Astronomy, 105-24, California Institute of \\ Technology, Pasadena, CA 91125 ejb@astro.caltech.edu
}

\begin{abstract}
Summary. The association of $\gamma$-ray bursts (GRBs) and core-collapse supernovae (SNe) of Type Ib and Ic was motivated by the detection of SN $1998 \mathrm{bw}$ in the error box of GRB 980425 and the now-secure identification of a SN 1998bw-like event in the cosmological GRB 030329. The bright radio emission from SN 1998bw indicated that it possessed some of the unique attributes expected of GRBs, namely a large reservoir of energy in (mildly) relativistic ejecta and variable energy input. Here we discuss the results of a systematic program of radio observations of most reported Type Ib/c SNe accessible to the Very Large Array, designed to determine the fraction of Type Ib/c SNe driven by an engine. We conclude that: (i) the incidence of such events is low, $<3 \%$, and (ii) there appears to be a clear dichotomy between the majority of hydrodynamic explosions ( $\mathrm{SNe}$ ) and engine-driven explosions (GRBs).
\end{abstract}

\section{Hydrodynamic vs. Engine Driven Explosions}

Stellar explosions can be characterized by their kinetic energy, $E_{K}$, and the mass of the ejecta, $M_{\mathrm{ej}}$. Equivalently one may consider $E_{K}$ and the mean initial speed of ejecta, $v_{0}$, or the Lorentz factor, $\Gamma_{0}=\left[1-\beta_{0}^{2}\right]^{-1 / 2}$, where $\beta_{0}=v_{0} / c$. In this context, supernovae (SNe) and $\gamma$-ray bursts (GRBs), are distinguished by their ejecta velocities: $v_{0} \sim 10^{4} \mathrm{~km} \mathrm{~s}^{-1}$ as inferred from optical absorption features (e.g. 1), and $\Gamma_{0}>100$, inferred from the nonthermal prompt emission [2, 3], respectively.

In the conventional interpretation, $M_{\mathrm{ej}}$ for $\mathrm{SNe}$ is large because $E_{K}$ is derived from the (essentially) symmetrical collapse of the core and the energy thus couples to all the mass left after the formation of the compact object.

GRB models, on the other hand, appeal to an engine - a stellar mass black hole, which accretes matter on many dynamical timescales and powers relativistic jets (the so-called collapsar model; 4]). Observationally, this model is supported by the complex temporal profiles and long duration of GRBs, their high Lorentz factors, a high degree of asymmetry [5], and episodes of energy injection. 


\section{SN 1998bw: An Engine Driven Supernova}

The unusual SN 1998bw shares some of the unique attributes expected of GRBs. This Type Ic SN coincided in time and position with GRB 980425 [6], for which the inferred isotropic energy in $\gamma$-rays was only $8 \times 10^{47} \mathrm{erg}$ [7], three to six orders of magnitude fainter than typical GRBs. More importantly, SN 1998bw exhibited unusually bright radio emission indicating about $10^{50} \mathrm{erg}$ of mildly relativistic ejecta as well as variable energy input [8]. To date these features have not been seen in any other nearby SN. Thus, the empirical data strongly favor an engine in SN $1998 \mathrm{bw}$.

Two scenarios for the origin of SN $1998 \mathrm{bw}$ and its relation to GRBs have been proposed: (i) GRB 980425 may have been a typical burst but viewed well away from the jet axis (hereafter, the off-axis model), and (ii) SN 1998bw represents a different class of SNe.

A powerful discriminant between these two scenarios is the expected rate of SN 1998bw-like events. In the off-axis model, the fraction of Type Ib/c SNe that are powered by a central engine is linked to the mean beaming factor of GRBs, $f_{b}$ (e.g. [5]); a recent estimate is $\left\langle f_{b}^{-1}\right\rangle \sim 500$ [5]. Coupled with an estimated local GRB rate of $\sim 0.5 \mathrm{Gpc}^{-3} \mathrm{yr}^{-1}$ [9] compared to a Type Ib/c $\mathrm{SN}$ rate of $\sim 4.8 \times 10^{4} \mathrm{Gpc}^{-3} \mathrm{yr}^{-1}$ [10, 11, 12], we expect that $\sim 0.5 \%$ of Type Ib/c SNe will be similar to SN 1998bw.

On the other hand, if SN 1998bw is not an off-axis burst, then the rate of similar events has to be assessed independent of the GRB rate. In this context, Norris (2002) [13] has argued that of the 1429 long-duration BATSE bursts, about 90 events possess similar high-energy attributes as that of GRB 980425. This number corresponds to about 25\% of Type Ib/c SNe within $100 \mathrm{Mpc}$.

\section{A VLA Survey of Type Ib/c Supernovae}

Our basic hypothesis is that (mildly) relativistic ejecta are best probed by radio observations, as was demonstrated in the case of SN 1998bw. To this end we began a program of observing most reported Type Ib/c SNe with the Very Large Array in late 1999 [14.

Figure 1 provides a succinct summary of the radio lightcurves and upper limits. Three strong conclusions can be drawn from this Figure. First, SNe as bright as SN 1998bw are rare; we find a limit of $<3 \%$ from our survey. Second, there is significant dispersion in the luminosities of Type Ib/c SNe. Finally, the radio emission from SNe (including SN 1998bw) is orders of magnitude dimmer than that of GRB afterglows.

\subsection{Expansion Velocities and Energetics}

In the framework of synchrotron self-absorption, the peak time and peak luminosity directly measure the mean expansion speed of the fastest ejecta [17. 


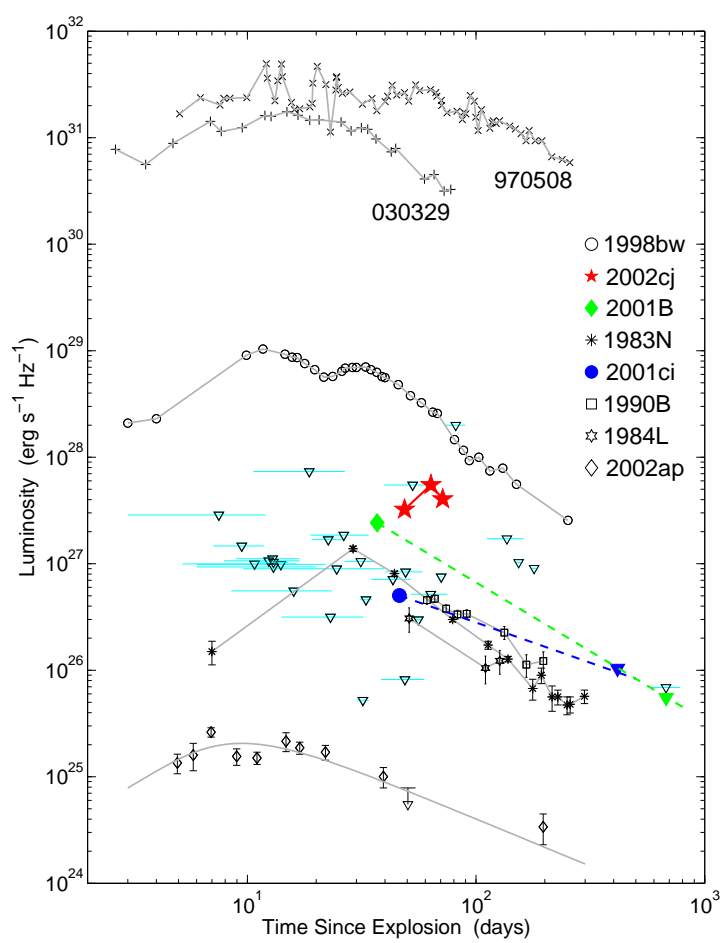

Fig. 1. Radio lightcurves of Type $\mathrm{Ib} / \mathrm{c}$ SNe detected in this survey and from the literature, as well as upper limits for the non-detections (Ref. 14 and references therein). We also include the radio lightcurves of GRB 970508 [15] and GRB 030329 [16. The uncertainty in time for the non-detections represents the uncertain time of explosion.

We infer velocities ranging from $v \sim 10^{4}$ to $10^{5} \mathrm{~km} \mathrm{~s}^{-1}$ based on our detections and upper limits 14 .

We also find that the ejecta giving rise to the radio emission from $\mathrm{SNe}$ for which detailed information is available (SN 1984L and SN 2002ap) can be produced by a hydrodynamic explosion [18, 14]. In fact, the estimated energies from the hydrodynamic models [19, 20, 21] exceed those inferred from the radio observations by up to two orders of magnitude. This may indicate that the total kinetic energies have been over-estimated, possibly as a result of neglecting a mild asymmetry.

We therefore conclude that none of the SNe observed in our survey and in the past clearly exhibits the unique characteristics of SN 1998bw: a significant excess of energy in mildly relativistic ejecta. 


\section{A Comparison to $\gamma$-Ray Burst Afterglows}

From Figures 1 and 2 it is clear that the radio lightcurves of GRB afterglows and SNe are dramatically different. This has significant implications, namely none of the Type Ib/c SNe presented in Figure 1 could have given rise to a typical $\gamma$-ray burst. However, SN $1998 \mathrm{bw}$ is unique in both samples: it is fainter than typical radio afterglows of GRBs but much brighter than Type $\mathrm{Ib} / \mathrm{c}$ SNe (Figure 2).

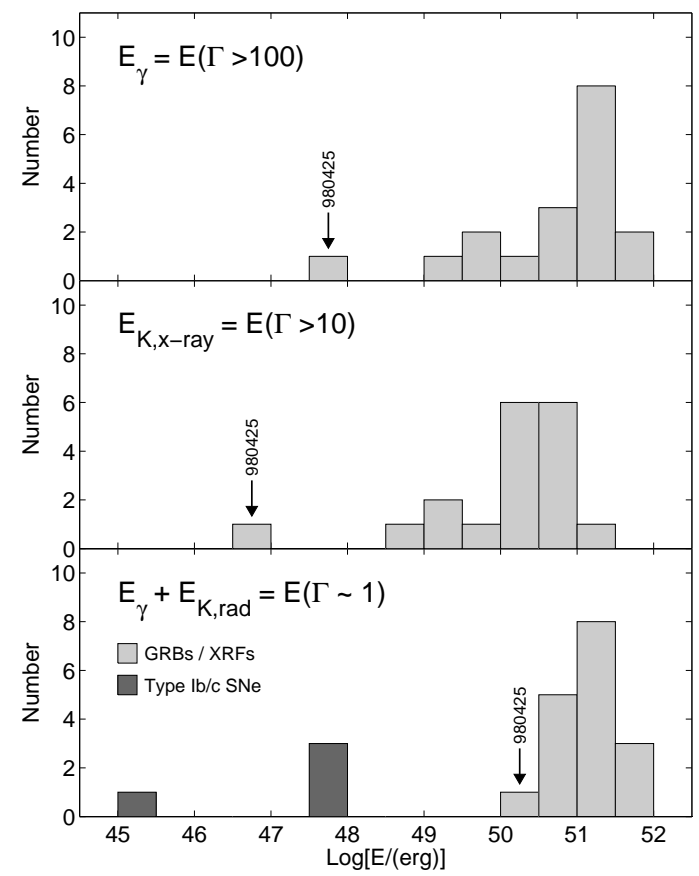

Fig. 2. Histograms of the beaming-corrected $\gamma$-ray energy $\left[22, E_{\gamma}\right.$, the kinetic energy inferred from X-rays at $t=10 \mathrm{hr}$ [23], $E_{K, X}$, and total relativistic energy, $E_{\gamma}+E_{K}$, where $E_{K}$ is the beaming-corrected kinetic energy inferred from the broadband afterglows of GRBs 80 24] and radio observations of SNe. The wider dispersion in $E_{\gamma}$ and $E_{K, X}$ compared to the total energy indicates that engines in cosmic explosions produce approximately the same quantity of energy, thus pointing to a common origin, but the ultra-relativistic output of these engines varies widely. In Type Ib/c SNe, on the other hand, the total explosive yield in fast ejecta (typically $\sim 0.3 c)$ is significantly lower.

\subsection{Hypernovae}

The discovery of broad optical lines and large explosive energy release, > few FOE, in SN 1998bw prompted some astronomers to use the designation 
"hypernovae" for SN 1998bw-like SNe. Unfortunately, this designation is not well defined, and has been applied liberally in recent years.

In our framework the critical distinction between an ordinary supernova and a GRB explosion is relativistic ejecta carrying a considerable amount of energy. Such ejecta are simply not traced by optical spectroscopy. This reasoning is best supported by the fact that the energy carried by the fast ejecta in SN 1998bw and SN 2002ap [18] differ by four orders of magnitude even though both exhibit broad spectral features at early times and both have been called hypernovae.

\section{Conclusions}

We end with the following conclusions. First, radio observations provide a robust way of measuring the quantity of energy associated with high velocity ejecta. This allows us to clearly discriminate between engine-driven SNe such as SN 1998bw and ordinary SNe, powered by a hydrodynamic explosion, such as SN 2002ap [18. Second, at least $97 \%$ of local Type Ib/c SNe are not powered by engines and furthermore have a total explosive yield of only $10^{48} \mathrm{erg}$ in fast ejecta. As summarized in Figure 2 this indicates that there is a clear dichotomy between Type $\mathrm{Ib} / \mathrm{c} \mathrm{SNe}$ and cosmic, engine-driven explosions.

\section{References}

1. Filippenko, A. V. 1997, ARA\&A, 35, 309.

2. Goodman, J. 1986, ApJ, 308, L47.

3. Paczynski, B. 1986, ApJ, 308, L43.

4. Woosley, S. E. 1993, ApJ, 405, 273.

5. Frail, D. A. et al. 2001, ApJ, 562, L55.

6. Galama, T. J. et al. 1998, Nature, 395, 670.

7. Pian, E. et al. 2000, ApJ, 536, 778.

8. Li, Z. and Chevalier, R. A. 1999, ApJ, 526, 716.

9. Schmidt, M. 2001, ApJ, 552, 36.

10. Marzke, R. O., et al. 1998, ApJ, 503, 617.

11. Cappellaro, E., Evans, R., and Turatto, M. 1999, A\&A, 351, 459.

12. Folkes, S. et al. 1999, MNRAS, 308, 459.

13. Norris, J. P. 2002, ApJ, 579, 386.

14. Berger, E. et al. 2003, ApJ in press; astro-ph/0307228

15. Frail, D. A., Waxman, E., and Kulkarni, S. R. 2000, ApJ, 537, 191.

16. Berger, E. et al. 2003, Nature in press; astro-ph/0308187

17. Chevalier, R. A. 1998, ApJ, 499, 810.

18. Berger, E., Kulkarni, S. R., and Chevalier, R. A. 2002, ApJ, 577, L5.

19. Chevalier, R. A. 1982, ApJ, 258, 790.

20. Matzner, C. D. and McKee, C. F. 1999, ApJ, 510, 379.

21. Iwamoto, K. et al. 2000, ApJ, 534, 660.

22. Bloom, J. S., Frail, D. A., and Kulkarni, S. R. 2003, astro-ph/0302210

23. Berger, E., Kulkarni, S. R., and Frail, D. A. 2003, ApJ, 590, 379.

24. Panaitescu, A. and Kumar, P. 2002, ApJ, 571, 779. 